\title{
SICARDIS, SENYORA DE MONTSENY I DE LLORET
}

\author{
J.M. Pons i Guri
}

La noble dona Sicardis era filla d'Amat, que fou vescomte de Girona, i d'altra Sicardis, tots dos difunts abans de l'any 1032. Per qüestions patrimonials sorgiren conflictes entre Sicardis i la seva mitja germana Ermesenda, primogenita del primer matrimoni d'Amat, i amb el marit d'aquesta, Guerau, vescomte de Girona.

Es mirà de resoldre la contenció per mitjà d'un conveni, en virtut del qual Sicardis restaria amb l'alou de Lloret $i$ els que tenia a l'Estanyol i les Croses $^{\prime} i$ havia de renunciar al patrimoni restant rebut dels seus pares, tant en llocs com en alous. Gestionava els interessos de Sicardis el qui documents posteriors ens presenten com a marit d'aquesta, Gaucfred Bernat. L'acord es formalitzà per escrit el 10 de gener de 1032, entre Gaucfred i els vescomtes de Girona, i es va pactar que Sicardis atorgaria la renúncia dins del termini que aniria des del vinent Carnestoltes ( 13 de febrer) i el de l'any següent (4 de març de 1033); en garantia del seu compliment, Gaucfred Bernat lliurava l'alou de Lloret en penyora, de tal manera que si la renúncia no es formalitzava dins del termini fixat 1 'alou romandria definitivament per als vescomtes i llur fill Bernat ${ }^{2}$.

Per raons que no coneixem, Sicardis no va donar compliment al que s'havia compromès Gaucfred i l'alou de Lloret fou guanyat pels vescomtes Guerau i Ermesenda.

Deu anys després, Sicardis, ja en majoria d'edat i ostentant l'apel-latiu de femina, propi aleshores de les dames d'alta condició, el dia 23 de gener de 1041 arribà a un bon enteniment amb els vescomtes de Girona, que

1' L'any 1002 els comtes de Barcelona Ramon Borrell i Ermesenda desmembraren aquest alou de Lloret del que aleshores era l'immens terme de Maçanet il'empenyoraren al vescomte Seniofred de Girona (J.M. PONS I GURI, «Document del vescomte Seniofred de Gironas, Recull d'Estudis d'Història Juridica Catalana, vol. III, Barcelona 1989, p. 17-30). Des d'aleshores pertanyé a la casa vescomtal de Girona i als seus descendents, fins a la mort de Sicardis. L'alou de l'Estanyol havia de ser molt gran, com aixi ho fan veure els masos que s' hi anomenen i les segregacions que anà sofrint.

${ }^{2}$ Apendix 1 . 
l'instrument tipificà com una comutatio o permutació, en virtut de la qual renunciava definitivament al patrimoni rebut dels seus pares - excepte els alous de l'Estanyol-i rebia, en canvi, l'alou de Lloret'.

Sicardis, aleshores senyora de Lloret i vídua de Gaucfred Bernat, del qui havia tingut quatre fills: Ermesenda, Guisla, Arbert i Bernat Gaucfred, va contreure segon matrimoni amb Umbert Odó, senyor dels castells de les Agudes i de Montpalau. Això esdevindria abans de l'any 1045, car el 9 de febrer d'aquest mateix any, en el placitum presidit per Umbert Odo amb el seu jutge Guifred, sobre les pertinences del monestir de Sant Cugat a la vall de Gualba, també hi és present Sicardis uxor eius ${ }^{4}$.

El segon marit de Sicardis, Umbert Odó, era fill d'Odó Vivas, conegut per Ot de les Agudes, que, en vida, havia estat un dels primats del comte de Berenguer Ramon I $i$ de la comtessa vídua Ermesenda'; la seva mare, Gerberga femina, fou protectora del monestir de Sant Marçal de Montseny, radicat a la seva dominicatura ${ }^{6}$. Malgrat que en aquesta etapa històrica la documentació és relativament escassa, els esments al noble Umbert Odó són quelcom freqüent: forma part del seguici de la comtessa àvia Ermesen$\mathrm{da}^{7}$, és amic i marmessor testamentari d'aquesta ${ }^{8}$ i figura entre els magnats de la cohort de Ramon Berenguer I i Almodis?. A més del placitum

${ }^{3}$ Apèndix II.

${ }_{4}^{4}$ Arxiu de la Corona d'A ragó (que d'ara endavant designem per ACA), Cartoral de Sant Cugat del Vallès, f. 319, n. 954).

52 de setembre de 1019 ....supranominata Ermesinda comitissa ordinante, assentiente filio suo domno Berengario marchione comite, una cum primatibus palatii sui [...] Odo Acutensi... (Arxiu Capitular de Barcelona, Liber Antiquitatum I, f. 151, n. 396).

6 ACA. Monacals d'Hisenda. Sant Marçal de Montseny. Pergamins en curs de catalogació. 6 d'octubre de 1057, donació de Gerberga femina, vídua d'Odó, al monestir de Sant Marçal de Montseny, d'alous a la vali d'Arbucies.

7 'L'any 1041. present amb la comtessa Ermesenda al lliurament del castell de Benedormiens a l'abat de Sant Feliu (Marca Hispanica, ap. 223). Any 1052, en l'elecció de l'abat de Sant Feliu de Guíxols (J. Vm. AnUeva, Viaje Literario a las Iglesias de España, XV, ap. L.). Any 1053, present en la venda que fa la comtessa a utilitat del monestir de Sant Pol (J.M. PoNs I GuRI, Diplomatari del monestir de Sant Pol de Mar, doc. 12, en Recull cit.).

${ }^{8}$ El 25 de setembre de 1057, rep també una deixa testamentària de la comtessa (P. DE BofaruL, Los Condes de Barcelona vindicados, II, p. 51-54).

${ }^{9} \mathrm{El} 12$ de novembre de 1056 , és present a l'atorgament de l'esponsalici de la comtessa Almodis (Marca Hispánica, ap. 246). Esmentat l'any 1057 entre els nobilibus viris dels comtes Ramon Berenguer i Almodis (ACA, Pergamins Ramon Berenguer I, n. 203). En 1058 , en la concèrdia dels comtes de Barcelona i Besalú amb el bisbe de Vic sobre el castell de Colltort (ACA, Trasllats de Ribera, II, p. 207). En 1059 forma part del tribunal que decideix sobre els greuges comesos per Mir Geribert (A. Rovira t Virgit.1, Història Nacional de Catalunya, vol. III, p. 499). En 1061 és present en la concòrdia de Ramon Berenguer I amb el vescomte Ponç Guerau sobre l'església de Celrà (F. MıQuel, Liber Feudorum maior, doc. 403). En 1062, ho és en el conveni del comte de Barcelona amb Guerau Alamany sobre el castell de Montagut (L. Feudorum cit., núm. 289) i també en la del mateix comte amb Ramon Bernat de Peratallada sobre el castell de Begur (ibid. núm. 472). En 1063 signa en l'acord entre Ramon Berenguer I i els germans Ricolf sobre el castell de Balsareny (ibíd. núm. 175). 
anteriorment esmentat de l'any 1045, l'any 1063 presideix amb la seva muller Sicardis un altre iudicatum celebrat a Sant Pol de Mar ${ }^{10}$; i el 1065 , amb la muller i els fills, és la figura principal en l'acte d'entronització de l'abat de Sant Marçal de Montseny ${ }^{\text {". }}$. En el discutit $\mathrm{i}$ indatable usatge Haec sunt usualia, hi figura present com a Umbertus de ipsis Acutis, entre altres magnats de la terra.

Sembla que Umbert Odó havia mort abans de l'any 1071, quan veiem que el seu lloc a la cort comtal l'ocupa el seu fill Guillem Umbert ${ }^{12}$. El patrimoni d'Umbert d'Odó era important, car, a més de disposar de la jurisdicció omnímoda als termes dels castells de les Agudes i Miravalls en la conca interior del riu Tordera, tenia la del castell de Montpalau a la banda sud-oest de la marina del comtat de Girona, bé que, quant a aquest darrer castell, estigués obligat a retre'n la potestat quan li fos demanada pel comte de Barcelona ${ }^{13}$.

A la mort d'Umbert Odó, el seu fill Guillem Umbert rebé els castells amb els seus termes i la part més considerable del patrimoni paternal. A Sicardis i als altres fills d'Umbert Odó, els restà del patrimoni paternal alous diversos, principalment a la marina.

Des d'aleshores, Sicardis fixà la seva residència principal a Lloret, on s'havia bastit un castell, convivint amb els fills tinguts dels dos matrimonis: Guisla, Arbert i Bernat Gaucfred, Ramon Umbert, Udalard i el jove clergue Bernat Umbert, que, amb el temps, esdevingué bisbe de Girona.

El 8 de gener del 1079, es va consagrar l'església parroquial de Sant Romà de Lloret, i Sicardis, amb els seus fills Bernat i Udalard, la doten dels terrenys que formaran la seva sagrera ${ }^{14}$. Pocs dies després, el dia 23 del mateix mes, doten la capella de Sant Joan, acabada de consagrar pel bisbe Berenguer Guifré de Girona, del seu castell de Lloret, participant-hi també el seu castla Odo ${ }^{15}$. Com que Sicardis, en els béns que foren d'Umbert Odó, hi té el seu decimum o esponsalici, participa en diversos atorgaments de l'hereu Guillem Umbert, com esdevé l'any 1083 en el conveni amb l'abat de Sant Cugat del Vallès sobre possessions de Gualba, Palautordera, Vall-

En 1064 participa en l'acord entre Guillem Bernat d'Odena i Albert Bernat sobre el castell d'Arrahona (ibid, núm. 471) i, a part, com a marmessor, juntament amb la comtessa Almodis, del capiscol Ponç de Girona (J. Ruus, Cartes antigues de Sant Marti Sacosta, en Analecta Sacra Tarraconensia, vol. IV, p. 17). En 1066 és testimoni en la venda de la Torre de Dela que fa Arbert Berenguer al comte de Barcelona (Lib. Feudonam, cit. núm. 315).

${ }^{10}$ Diplomatari del Monestir de Sant Pol de Mar, cit. doc. 16.

II VIL LanueVA, Viage, cit. VI, ap. XXXIV.

12 Poc abans de 1071 (ACA, Pergamins Ramon Berenguer I, núm. 9).

13 Document indatat. Lib. Feudorum, cit. núm. 384.

14 J.M. Ponsi GuRI, «Dotalies d'esglésies selvatanes del bisbat de Gironas, Recull, cit. II, p. 472.

15 Dotalies, cit. p. 474. 
gorguina i Pertegaç ${ }^{16}$, i dóna consell a l'esmentat hereu en la donació de la capella de Sant Celoni, que l'any 1088 fa a favor del monestir de Santa Maria de l'Estany ${ }^{17}$. En un conjunt de diversos documents, atorgats en la seva viduïtat ${ }^{18}$, el més recent és de l'any $1096^{19}$.

Sicardis arribà a una edat molt avançada. Recordem que en l'any 1033 figura com a persona vivent $\mathrm{i}$ amb drets i que en l'any 1041 l'hem vista atorgant com a sui iuris $\mathrm{i}$ sense que ningú completi la seva personalitat jurídica, ço que ens porta a suposar-li una edat de prop de vint anys; tampoc podem oblidar que abans de 1045 ja havia tingut, pel cap baix, els quatre fills que li coneixem del seu matrimoni amb Gaucfred Bernat. Per tant, podem deduir facilment que, a la seva mort, esdevinguda, com veurem a continuació, en el trencall dels anys 1103 i 1104, hauria ultrapassat els vuitanta anys, havent sobreviscut als seus dos marits i als seus fills Guisla i Arbert, del primer matrimoni, i Ramon, Udalard i Guillem Umbert, del segon.

El 6 d'octubre de 1103, trobant-se malalta en el seu castell de Lloret, va atorgar testament per escrit $i$ el signà amb la seva mà, en presència de sis testimonis $\mathrm{i}$ un arxiprest en funció d'escrivà ${ }^{20}$. És un testament com els altres atorgats sota l'imperi de les lleis gòtiques, sense institucio d'hereu pròiament dita. Elegeix els acostumats marmessors o almoiners, disposa el seu enterrament al cementiri de la Seu de Girona i fa deixes al seu capitol canonical de terres a l'Estanyol, a Lloret $\mathrm{i}$ altres indrets; confirma una donació feta al monestir de Sant Marçal i una altra a la capella de Sant Celoni; fa altres llegats a l'església de Sant Julià de Montseny i a l'Hospital de Girona. Deixa un mas, també a l'Estanyol, al marmessor Ponç Odó, tal volta familiar; un altre mas a Vilardell, a una néta anomenada Sicardis; i un altre a Maçanet de la Selva a la seva filla Ermesenda. Semblen els més afavorits els seus filis Bernat Gaucfred i Bernat Umbert, que aleshores era bisbe de Girona, als qui dóna en indivís un altre alou a l'Estanyol i el castell de Lloret amb totes les seves pertinences, amb el benentès que Bernat Umbert tindria l'usdefruit de la meitat del castell, el qual a la seva mort, passaria a Bernat Gaucfred i si aquest moria, al fill que deixés, passant l'altra meitat del castell de Lloret a la canònica de Girona. Quant als altres béns, els que procedien de la banda del seu primer marit, incloent-hi els que a títol de luctuosa li havien pervingut dels seus fills de primer matrimoni, Guisla i Arbert, passaven a Bernat Gaucfred; en canvi, els existents a la

16 ACA, Cartoral de Sant Cugat, núm. 976.

17 J.M. Pons I GURI, «Un cartoral de la Milícia Hospitalària», Recull, eit. III, p. 416-418.

18 Entre altres, any 1084, permuta d'alou amb el monestir de Sant Pol (Diplomatari, cit. doc. 21), any 1090, definició d'alou a Pineda (ibíd., doc. 22) i any 1094, definició d'un molí (ibíd., doc. 25).

${ }^{19}$ ACA, Cartoral de Sant Cugat, núm. 760.

${ }^{20}$ Apèndix III. 
marina o terme del castell de Montpalau i llocs veïns, procedents del que havia rebut del seu marit Umbert Odo, adhuc el decimum o esponsalici encara no rebut, passaven al bisbe Bernat Umbert.

No coneixem amb precisió la data de la defunció de Sicardis, però consta la publicació del testament escrit celebrada amb intervenció judicial a l'altar de Santa Anastàsia de la Seu de Girona el 19 de febrer de 1104, dins del temps legal $\mathrm{i}$ amb la formalitat establerta segons el primer mode de la llei Morientium scripta voluntas del Liber Iudicum ${ }^{21}$.

Poques vegades hom pot disposar simultàniament de l'exemplar escrit d'un testament $i$ de la seva publicació sagramental. En el nostre cas és així, bé que, dels respectius diplomes, el testament és un trasllat i les conditiones de la publicació són en origínal.

La publicació recensiona gairebé literalment el contingut de les parts dispositives del testament escrit, en el qual en la clàusula que conté les substitucions referents al castell de Lloret hi interpola el que es podria entendre per aclariment o interpretació, amb les paraules ...qui canonicus sit Gerunde. Post obitum autem predicti filii Bernardi Gaucefredi qui sit canonicus Gerunde... que no figuren en el testament escrit. Es pot donar també el cas que, tractant-se el testament d'un trasllat de l'original, l'escrivà que l'havia transcrit hagués omès involuntàriament aquelles paraules, que mantindria el text de la publicació.

21 Apèndix IV. A més del diploma original que transcrivim, al mateix Arxiu de la Catedral de Girona hi ha un trasllat coetani en el pergamí núm. 135, carpeta 4, que no presenta cap variació. 


\section{ANNEX}

\section{I}

1032, gener 10

Empenyorament de l'alou de Lloret, atorgat per Gaufred Bernat a favor dels vescomtes de Girona, Guerau i Ermessendis, i llur fill Bernat.

Arxiu Capitular de Girona, perg. 40. (Trasllat coetani.)

In nomine Domini. Ego Gaufredus Bemardi inpignorator sum uobis Gerallo uicecomite et uxor sua Ermessendis uicecomitissa, et filio uestro Bernardo si uos mortui eritis ad terminum subtus scriptum, ipsum meum alaude quod ego abeo in Comitatu Gerundense, in locum que dicunt Lauredo in terminio Macianeto. Et est ipsum alaudem chasas cum curtes, et chasalis, et ortis, ortalis, et uineas et uinealis et eras cultas et eremas, cum omnia genera arborum, et siluis et garricis, montibus et uallibus, excelsus et pronum; et aduenit predictum alaudem per parentorum meorum uel per mea comparacione, siue per qualicumque uoce; et affrontat iam dicto alaude de parte orientis intus in Tursa uel in eius termines, de meridie intus in ipsa mare, et de occiduo in ipso uillare uel in eius termines; de parte uero circii affrontat in uico que nuncupant Challes uel in eius termine. Quantum infra istas. iiii $^{\text {or }}$, affrontaciones includunt sic impignoro uobis predictum alaudem de Lauredo, in tali uidelicet raciones uel conuentu ut Sicardis filia de Amatus condam et de Sicardis condam faciat talem securitatem aut perdonacionem aut per qualicumque cessionem de omnem suam hereditatem quod abet uel abere debet in omnibus locis et in omnibus alaudis, tam in condirectis quam in eremis, tam in excelsis quam in pronis, tam in aquis quam in petris, condirectis quam in eremis que fuerunt de genitori uel de genitrice predicti, et hanc securitatem faciat in potestate de Gerallo predicto uel de Ermessendis predicta uel de Bernardo predicto et talem faciat hunc securitatem uel cessionem unde legaliter tenere uel abere possint predictus Gerallus uel uxor sua predicta uel predictus filius eius omnem suam hereditatem suprascriptam exceptus ipsum alaudem de Stagnolo uel de ipsas Crosas de Amatus et Sicardis ibi abebant ipsa die quando migrauerunt ab hoc seculo. Et hanc securitatem uel cessionem fiat factam de istas Carnestoltas usque ad alias primas aduenientes et fiat tradita in potestate de predicto Gerallo uel de uxor sua predicta uel de predicto filio eius sine illorum enganno. Et si hoc factum non fuerit a predictum terminum, incurrat predictum alaudem de diam dicto Lauredo cum suis terminis in potestate de Gerallus uel de uxore uel de filio eius a proprium ad facere quod uoluerint sine cuiusdam contradiccione aut minoracione et malo ingenio uel occasione. Quod si ego Gaufredus Bernardi aut ullusque uiuens homo utriusque sexus uenero aut uenerit ad inrumpendum hunc sacriptura impignoracionis, non hoc ualem aut ualeant uendicare sed componam aut componant predictum alaudem de Lauredo in duplo cum omnem suam melioracionem et in antea ista scriptura impignoraciones firma permaneat modo uel omnique tempore. Facta ista scriptura impignoracione. III $^{\circ}$. idus ianuarii anno XXXVI. quibus dicitur obisse Rotbertus rex qui et primus 
tempus regnare Aianricus filius eius. Sigænum Gaufredus Bernardi prolis, qui hunc impignoracione fieri iussít et scribi et manu sua firmauit et testibus firmare rogauit. Sigศnum Remundo Uitale. Sigশึum Guillelmus Vitalus. Sigđnum Amatus Rotfredus.

Et ego Gaucfredus suprascriptus ad istis suprascriptis terminis abuero, facta ista carta et tradita per manus de prescripta Sicardis in potestate de predictus Geraldus uel de uxor predicta uel de filio eius, si uos suprascripta recipere uultis sicut superius scriptum est.

(Senyal) Udamarius presbiter qui ista scriptura inpignoraciones scripsit is die et anno quo supra, et cum literis superpositas in uerso. $1^{\circ}$. uel. $n^{\circ}$. et rasas uel emendatas in uerso. $\mathrm{Xv}^{\circ}$, et 1 .

Ego Petrus qui hoc transtuli.

\section{II}

1041, gener 23 .

Permutació atorgada pels vescomtes de Girona, Guerau i Ermesenda, amb la noble Sicardis, per la qual aquesta rep l'alou i castell de Lloret i aquells l'alou de l'Estanyol.

Arxiu Capitular de Girona, pergamí 47. (Trasllat coetani.)

In Christi nomine ego Guirallus uice comite et Ermessendis uxoris mee uicecomitissa, comutatores sumus tibi Sicardis femina. Manifestum est enim quia placuit animis nostris et placet nullius quoientis imperio nec suadentis ingenio, sed propria expontanea hoc elegit nobis bona uolumptas ut scripturam comutacionis fecissemus tibi Sicardis femina sicuti et facimus. Donamus namque tibi predicta Sicardis in comutacione ipsum alodem de Laured, cum omnibus terminis et affrontacionibus suis et pertinenciis et aiagenciis suis, quantum fuit de Gaufredi Bernardi que est condam, et aduenit nobis Guirallo et Emessendis comitatoribus supra scriptum alodem per scripturam impignoracionis et conuentum quod nobis fecit predictus Gaucefredus et minime nobis compleuit, et propter hoc supra scriptum alodem in nostra potestate ad proprium alodem (per)uenit. Est namque suprascriptum alodem in comitatu gerundense in locum que dicitur Laurede. Affrontat namque predictum alodium de parte orientis in terminio de Torsa et de meridie in mare. De parte uero circi affrontat in Monte Barbad et in Calldes, et de occiduo affrontat in puio de Gerundella uel in termines de Blannes. Quantum infra istas, $\mathrm{um}^{\text {or }}$, affrontaciones includunt, sic donamus ego predictus Guirallus et Ermessendis a te iamdicta Sicardis in comutacione, quantum nobis pignorauit predictus Gaucefredus sicut in ipsa scriptura resonat quod ille nobis fecit, et accepimus ego predictus Guirallus et Ermessendis de te predicta Sicardis in comutacionem omnem hereditatem quod habebas uel habere debebas et te contingit uel contingere debet per omnibus modis per uocis genitoris tui Amati et ienitricis tue Sicardis quantum habes uel habere debes de supra scripta omnia hereditate in comitatu Gerundense et Barchinonense, exceptus ipso alodem de Stagnolo, quod tu Sicardis libenti animo suprascriptam tuam hereditatem nobis in cornutacionem dedisti per suprascriptum alodem de 
Laurede, quod nos in comutacionem donauimus et tu predicta Sicardis libenti animo suscepisti. Et si nos predicti comutatores Guirallus et Ermesindis contra hanc scriptura comutacionis uenerimus ad irrupendum uel ad inquietandum, aut ullus homo utriusque sexus propter uoces nostras, componamus aut componant supra scriptum alodem de Laurede in duplum cum omnem inmelioracionem suam, et in antea hec scriptura comutacionis nostre firma permaneat omnique tempore. Facta hec comutacio. X. kalendas februarii annos. X. regnante Angelrigus. Girallus uices comes (senyal). Sigrenum Ermessendis uicecomitissa, qui ista conmutacione firmauimus et testes fimare rogauimus. Ermengaudus leuita (senyal). Sigrnum Guillelmus de Todela. Siginum Amad. Sigtnum Dalmad. (Senyal) Seniofredi leuita.

(Senyal) Arnulfus presbiter que hanc scripturam comutacionis scripsi cum literas superpositas in uerso tercio et. XII. ubi dicit «simul» et «exceptus» die et anno quod supra.

(senyal) Bernardus leuita qui hoc translatum scripsi.

\section{III}

1103, octubre 6, Lloret de Mar

Testament escrit de Sicardis, senyora del castell de Lloret.

Arxiu Capitular de Girona, pergami 136. (Trasllat coetani.)

Quia nullus in carne positus mortem euadere potest ic circo ego Sicardis, timens repentine mortis interitum, plena ac sincera memoria, dum iaceo in domo mea apud castrum de Laured, eligo tutores meos uel elemosinarios Bernardum uidelicet filium meum Gerundensem Episcopum et Bernardum Gaucefredi fratres et Raimundum Geralli et Berengarium Otonis et Poncium fratrem eius, quibus plenam laudo atque concedo potestatem distribuendi omnes res meas, mobibiles et inmobiles, sicuti ego in presenti testamento disposuero. In primis dimitto corpus meum sepeliendum in cimiterio Sancte Marie Sedis Girunde, et canonice eiusdem ecclesie concedo apud Estaniol ipsum mansum de Oliuera et condaminam de ipso Crosa et ipsam quam tenet Raimundus de Pulcromiraculo, et apud Lauretum ipsum mansum de Campo Guardial cum ipsa francheda et tracturis et pertinentiis suis ubi habitat Poncius Miro, et in Ualle Marine ipsum alodium qui fuit Raimundi Umbert, et ipsum mansum de Trilia ubi <Isarnus> Ysarnus habitat cum omnibus suis pertinenciis et ipsam meam mulam et omne aesplet quod habeo in omni alodio quem teneo et dimitto pre libate canonice, dono et concedo predicte canonice; et Sancto Marciali superiori de Montesigno dimitto donationem quam ego feci ei apud Lauretum, et Sancto Celedonio ipsas comparationes quas ego feci intus in ipsa uilla Sancti Celedonii pro emendatione alaudi de Ual de Marina; et Poncio Otonis dimitto unum mansum apud Stagneolum qui est in Rexag quem tenet Amallus Guillelmi cum suis pertinentiis, et meam partem de ipsis equis quas habeo in Casellas et ipse mansus post obitum predicti predicto Poncii reuertatur ad filios meos Bernardum episcopum et Bemardum Gaucefredi, et alium meum alodium quod habeo apud Stagneolum infra parrochia Sancti Andree, excepta donatione quam ego Sancte Marie Uillebertrandi feci, dimitto iam dictis filiis meis Bernardo Ierundensi Episcopo et 
Bernardo Gaucefredi, cum omnibus baiuliis que ibi sunt. Et iterum concedo eis kastrum Laureti cum omnibus sibi pertinentibus, exceptis donationibus quas facio Canonice Gerundensi et Sancto Marciali, tali uidelicet ratione ut dum uixerit prefatus Bernardus episcopus teneat et possideat medietatem ipsi castri et omnium rerum eidem kastro pertinentium, absque blandimento ullius hominis; post obitum uero suum remaneat iam dicto Bernardo Gaucfredi fratri suo, et si Bernardus mortuus fuerit, uni filio suo remaneat medietas prefati kastri, et omnium rerum eidem kastro pertinentium predicte canonice Sancte Sancte Marie Girundensi sine blandimento ullius hominis uel femine sicut superius dictum est de Bernardo episcopo. Et omne meum quod habeo a Gaucfredo Bernardi uiro meo et luctuosos quos habeo de Guisla et Arberto fratribus, qui fuerint filii eius, et ipsa alodia que michi dedit Amatus Rutfredi per iussionem predicti Gaucfredi ubicumque sint dimitto Bernardo Gaucfredi per meliorationem. Et ad Ermessendem filiam meam dimitto mansum meum quem habeo in Macaned ad ipsas Brugeroles quem tenet Bonuspar, Et Sicardi nepte mee concedo ipsum mansum de Uilarzel. Alia uero alodia que fuerunt Gaucfredi Bernardi ubicumque sint, ab integro dimitto prefato Bemardo Gaucfredi filio meo alodia autem de Chaselles cum omnibus suis pertinenciis et ipsa de Perduds cum suis pertinentiis et ipsa de Ualle Mucusa cum suis pertinentiis, et ipsa de Capadaspre cum suis pertinentiis, et ipsa de Mata a maritimis cum suis pertinentiis, et kastrum de Kalella cum alodiis suis de Ortosauiniano et de Konte Sancto et de Cornesalter, et de Fonterotundo cum illorum pertinentiis et alodia de Ualleplana, dimitto Bernardo filio gerundensi episcopo; et iterum dimitto <ea〉 ei omnem meum decimum quod habere debeo de Umberto Otonis et omnes meas comparationes quas feci cum eo in omnibus locis, tam in Gerundensi quam etiam in Barchinonensi. Post obitum quoque suum dimitto Sancto Iuliano de Montesigno ipsum mansum de Uillare Tort. Et dimitto ad Ospitale Girundense dimidium modium ordei et quarta. i. de uino apud Stagneolum. Et de meo mobile exceptis uexellis sint soluta mea debita. Et ex aliis que remanent dimitto Petro Udalardi solidos, uii. de plata, et ex alio quod remanet dimitto medietatem Sancto Marciali superiori; aliam uero medietatem pro anima mea, sicuti melius uiderint mei elemosinarii. Actum est hoc. ii. nonas octobris anno. XI. III. regni Filippi regis. Sigtrum Sicardis que hoc testamentum fieri iussi, manu propria firmaui et testibus firmare rogaui. Siganum Raimundi Geralli. Sigwnum Berengarii Otonis. Sigønum Raimundi Berardi. Sigæenum Guillelmi Dominici. SigÆnum Iohannis Raimundi. * Poncius Otoni leuita.

Arnallus scriptis faieci archipresbiter istis, qui scripsit hoc die annoque prefixo (senyal) Guillelmus presbiter qui hoc fideliter translataui.

\section{IV}

\section{4, febrer 19, Girona}

Publicacio jurada del testament escrit atorgat per Sicardis, senyora de Lloret, el 6 d'octubre de 1103.

Arxiu Capitular de Girona, perg. 137. (Original.) 
Hee sunt condiciones sacramentorum facte ordinante Petro Bernardi iudice, coram sacerdotibus Berengario Guillelmi et Petro Suniarii, in presencia Arluini et Raimundi Stephani et aliorum hominum. Testificati sunt testes (lac. 8 spac.) his nominibus uocitati hi sunt: Arnaldus archipresbiter, Raimundus Guadamiri presbiter. Nos prefibati testes, unum dantes testimonium, iuramus in Deum et super altare sancte Anastasie quod situm est in ecclesia sancte Marie sedis Gerunde, quia nos uidimus et audiuimus et presentes ibi eramus quando quondam Sicardis femina, iacens in domu sua apud castrum de Laured, detenta ab egritudine qua obiit, dum adhuc esset in sua plena memoria ac loquela, ordinauit suam extremam uoluntatem et ordinationem suarum renum per suum firmum testamentum, per quod constituit ut essent sui elemosinarii et manumissores hi sunt: Bernardus Gerundensis Episcopus filius suus et Bernardus Gaucefredi fratres, et Raimundus Geralli ac Berengarius Otonis atque Poncius Otonis fratres, quibus iniuncxit distribuere omnia que habebat per ordinationem predicti testamenti, sicut hic scriptum est: Primum dimisit corpus suum sepeliendum in cimiterio Sancte Marie Sedis Gerunde, et concessit eiusdem ecclesie canonice apud Staniol ipsum mansum de Oliuera, et condemina de ipsa Crosa et ipsam quam tenet Raimundus de Pulcromiraculo, et apud Lauretum ipsum mansum de Campo Guardial, cum ipsa francheda et tracturis et pertinentiis suis ubi habitat Poncius Miro, et in Ualle Marina ipsum alodium qui fuit Raimundi Umberti et ipsum mansum de Trilia ubi Ysamus habitat, cum omnibus suis pertinentiis et ipsam suam mulam et omne esplet quod habebat in omni alodio quod tenebat, et dimisit prelibate canonice, donauit atque concessit predicte canonice, et Sancti Marciali superiori de Montesigno dimisit donationem quam ipsa fecerat ei apud Lauretum et Sancto Celedonio ipsas comparationes quas illa fecit intus in ipsa uilla Dancti Celedonii pro emendatione alodii de Ualle Marina. Et Poncio Otonis dimisit unum mansum apud Stagneolum qui est in Rexag, quem tenet Arnallus Guillelmi cum pertinentiis et suam partem de ipsis equis quas habebat apud Casellas; et ipse mansus post obitum predicti Poncii reuertatur ad filios suos Bernardum Umberti et Bernardum Gaucefredi; et alium de suum alodium quod habebat apud Stagneolum infra parroechia Sancti Andree, excepta donatione quam ipsa Sancte Marie de Uillebertrandi fecerat, et dimisit iam dictis filiis suis Bernardo Gerundensi Episcopo et Bernardo Gaucefredi cum omnibus baiuliis que ibi sunt. Et iterum concessit eis castrum Laureti cum omnibus sibi pertinentibus, exceptis donationibus quas fecerat Canonice Gerundensi et Sancto Marciali, tali uidelicet racione ut dum uixerit prefatus episcopus teneat et possideat medietatem ipsius castri et omnium rerum absque blandimento ullius uiuentis hominis; post obitum uero suum remaneat iamdicto Bernardo Gaucefredi fratri suo, et si Bernardus mortuus fuerit uni filio suo remaneat qui canonicus sit Genunde. Post obitum autem predicti filii Bernardi Gaucefredi qui sit canonicus Gerunde, remaneat medietas prefati castri et omnium rerum eidem castrum pertinentium predicte Canonice Sancte Marie Gerundensi sine blandimento ullius hominis uel femine, sicut superius dictum est de Bernardo episcopo. Et omnem suum decimum quod habebat de Gaucefredo Bernardi uiro suo et luctuosos quos habebat de Guisla et Arbertro fratribus qui fuerunt fifii eius et de ipsa alodia que ei dedit Amatus Ratfredi per iussionem predicti Gaucefredi ubicumque sint dimisit Bernardo Gaufredi per melioracione. Et ad Ermessendem filiam suam, dimisit mansum suum quem habebat in Macianeto ad ipsas Brugeroles quem tenet Bonuspar. Et Sicardi nepte mee concessit ipsum mansum de Uilarzel. Alia uero alodia que fuerunt Gaucfredi Bernardi ubicumque sint ab integro dimisit 
Bernardo prefato Gaucfredi filio suo. Alia autem de Kaselles cum omnibus suis pertinentiis et ipsa alia de Perduds cum suis pertinentiis et ipsa de Cantalobs et de Riba cum illorum pertinentiis, et ipsa de Vilar cum suis pertinentiis et ipsa de Ualle Mucuso cum suis pertinentiis et ipsa de Campodaspre cum suis pertinentiis etde Mata de maritimis cum suis pertinentiis et kastrum de Kalela cum alodiis de Ortosauiniano et de Monte Sancto et de Comes Altes et de Fonterotundo cum illorum pertinentiis et alodia de Uallplana dimisit Bernardo filio suo Gerundensi Episcopo, et iterum dimisit omne suum decimum quod habere debebat de Umberto Otonis et omnes suas comparationes quas fecit cum eo in omnibus locis, tam in Gerundensi quam etiam in Barchinonensi Post obitum quoque suum dimisit Sancto Iuliano de Montesigno ipsim mansum de Uillare Tort. Et dimisit ad Ospitalem Girundensem dimidium modium ordei et quarte. i. de uino apud Stagneolum. Et de mobile, exceptis uexellis, fuissent soluta sua debita. Et ex aliis que remanserunt dimisit Petro Udalardi solidos .vII de plata. Et ex alio quod remansit dimisit medietatem Sancti Marciali superiori. Aliam uero medietatem pro anima sua, sicuti melius uiderint sui elemosinarii. Fuit factum prescriptum testamentum. II. nonas octobris anno .XLIIII. regni Philippi regis. Firmatum fuit a supradicta conditrice Sicarde signo puncti et a reliquis firmatoribus, scilicet Raimundo Geralli et Berengarii Otonis, atque Raimundi Bernardi ac Guillelmo Dominici, necnon Iohannis Raimundi. Scriptor Arnallus archipresbiter. Nos supradicti testes testificamur et iuramus in Deum et in supradicto iuramento quaa, sicut superius scriptum est, item a supranominata testatrice Sicardi ordinatum est et firmatum nobis uidentibus et audientibus per extremam uoluntatem suarum rerum. Late conditiones .XI. kalendas marcii anno .XL.IIIL regni Philippi regis, infra primos .VI. menses post obitum predicte testatricis. Amallus scriptis fatet archipresbiter istis te

Raimundus Corauila secundus, qui supradictum testimonium dedimus et iurando firmauimus.

* Petrus Suniarii presbiter. Berengarius presbiter. Raimundus archileuita. Raimundi Stefani.

* Guillelmus ipodiaconus.

* Petrus caput scole atque iudex. 